\title{
Loss of Nuclear BAP1 Expression Is Associated with High WHO/ISUP Grade in Clear Cell Renal Cell Carcinoma
}

Young Chan Wi · Ahrim Moon ${ }^{1}$ Min Jung Jung ${ }^{1}$. Yeseul Kim Seong Sik Bang · Kiseok Jang Seung Sam Paik · Su-Jin Shin

Department of Pathology, Hanyang University College of Medicine, Seoul; 'Department of Pathology, Soonchunhyang University Bucheon Hospital, Soonchunhyang University College of Medicine, Bucheon, Korea

Received: July 13, 2018

Revised: August 24, 2018

Accepted: September 21, 2018

\section{Corresponding Author}

Su-Jin Shin, MD, PhD

Department of Pathology, Hanyang University

College of Medicine, 222-1 Wangsimni-ro,

Seongdong-gu, Seoul 04763, Korea

Tel: $+82-2-2220-8250$

Fax: $+82-2-2296-7502$

E-mail: charm@hanyang.ac.kr

\begin{abstract}
Background: BRCA1-associated protein 1 (BAP1) mutations are frequently reported in clear cell renal cell carcinoma (ccRCC); however, very few studies have evaluated the role of these mutations in other renal cell carcinoma (RCC) subtypes. Therefore, we analyzed BAP1 protein expression using immunohistochemistry in several RCC subtypes and assessed its relationship with clinicopathological characteristics of patients. Methods: BAP1 expression was immunohistochemically evaluated in tissue microarray blocks constructed from 371 samples of RCC collected from two medical institutions. BAP1 expression was evaluated based on the extent of nuclear staining in tumor cells, and no expression or expression in $<10 \%$ of tumor cells was defined as negative. Results: Loss of BAP1 expression was observed in ccRCC $(56 / 300,18.7 \%)$, chromophobe RCC (6/26, 23.1\%), and clear cell papillary RCC (1/4,25\%), while we failed to detect BAP1 expression loss in papillary RCC, acquired cystic disease-associated RCC, or collecting duct carcinoma. In ccRCC, loss of BAP1 expression was significantly associated with high World Health Organization (WHO)/International Society of Urological Pathology (ISUP) grade $(p=.002)$; however, no significant correlation was observed between loss of BAP1 expression and survival in ccRCC. Loss of BAP1 expression showed no association with prognostic factors in chromophobe RCC. Conclusions: Loss of BAP1 nuclear expression was observed in both ccRCC and chromophobe RCC. In addition, BAP1 expression loss was associated with poor prognostic factors such as high WHO/ISUP grade in cCRCC.
\end{abstract}

Key Words: Carcinoma, renal cell; Clear cell; BAP1; Immunostaining
Renal cell carcinoma (RCC) accounts for $2 \%-3 \%$ of all malignant diseases in adults. ${ }^{1}$ Clear cell renal cell carcinoma (ccRCC) is the most common renal tumor subtype and is closely associated with von Hippel Lindau (VHL) tumor suppressor gene mutations that lead to the stabilization of hypoxia-inducible factors in both sporadic and familial forms. Recently, three tumor suppressor gene mutations, namely, PBRM1, SETD2, and BAP1, located close to $V H L$ on chromosome 3 p were reported. ${ }^{2-4}$

Studies have reported BAP1 mutation in about $10 \%-15 \%$ of ccRCC cases. ${ }^{5,6}$ BRCA1-associated protein 1 (BAP1) is a nuclearlocalized deubiquitinating enzyme that was initially discovered as a BRCA1-associated protein and known to interact with multiple proteins. BAP1 was shown to exhibit a tumor suppressor role in several cancers through its deubiquitinase activity, thereby regulating target gene transcription, cell cycle control, DNA damage repair, and cellular differentiation. ${ }^{7}$ Inactivation mutations of the BAP1 gene, including insertion, deletion, frameshift, nonsense, and missense mutations, have also been reported. ${ }^{8}$
The germline mutation in the BAP1 gene is inherited in an autosomal dominant pattern. ${ }^{9}$ Affected individuals inherit a nonfunctional BAP1 allele, as observed with other tumor suppressors, and the remaining functional allele is inactivated later in life. There is a high risk for developing tumors, including atypical Spitz tumors, uveal melanoma, cutaneous melanoma, epithelioid malignant mesothelioma, and ccRCC. ${ }^{10}$ BAP1 germline mutations are associated with poor prognosis in uveal melanoma, cutaneous melanoma, and ccRCC. ${ }^{10}$ Sporadic BAP1 mutations have also been identified in several tumors, including uveal melanoma, ${ }^{11}$ malignant mesothelioma, ${ }^{12}$ and ccRCC. The loss of BAP1 expression in mesothelial cells in effusion cytology specimens is an indicator of possible mesothelioma. ${ }^{13}$ Nearly half of the investigated uveal melanoma tumors harbor an inactivating BAP1 mutation, which was strongly associated with the loss of BAP1 nuclear staining and other aggressive prognostic features. ${ }^{14}$ Furthermore, several studies have revealed the association between inactivating BAP1 mutation and high grade ccRCC, ${ }^{6}$ sarcomatoid 
transformation, and poor prognosis in patients with $\mathrm{cCRCC},{ }^{15}$ especially in those with low-grade RCC. ${ }^{16}$ The loss of BAP1 expression in immunohistochemical staining has been reported as a highly reliable method for the detection of BAP1 mutation. ${ }^{6}$ Although BAP1 mutations are frequently observed in ccRCC, limited data are available on the expression of BAP1 in other RCC types.

Therefore, we evaluated the loss of BAP1 nuclear expression in several subtypes of RCC, including ccRCC, papillary RCC, and chromophobe RCC, and analyzed its relationship with clinicopathological characteristics of patients.

\section{MATERIALS AND METHODS}

\section{Patient selection}

A total of 371 samples were retrospectively obtained from Hanyang University Hospital (247 cases, 2005-2017) and Soonchunhyang University Bucheon Hospital (124 cases, 20012013). Formalin-fixed, paraffin-embedded tissue samples obtained from surgically resected primary tumors at the time of initial diagnosis were collected. The pathologist in each institution reviewed the slides and selected a representative block for each case, and $3.0 \mathrm{~mm}$ of core tissue microarray (TMA) blocks were constructed, with two representative cores for each case. The patient and tumor characteristics, including age, type of surgery, histological type, histological grade, and follow-up data, were acquired. The histological subtypes were classified according to the 2016 World Health Organization (WHO) Tumor Classification. We graded ccRCC and papillary RCC according to the 2013 WHO/International Society of Urological Pathology (ISUP) grading system. ${ }^{17,18}$ Chromophobe RCC was graded according to the published parameters. ${ }^{19}$ All cases were reviewed by two pathologists for tumor type and WHO/ISUP grade. This study was approved by the Institutional Review Board of the Hanyang University Hospital (HYUH 2018-05-005), and the requirement for informed consent was waived.

\section{Immunohistochemistry for BAP1 expression}

Sections from the TMA blocks were immunostained using the Bond-max Automated immunohistochemistry (IHC)/in situ hybridization stainer (Leica Biosystems, Nussloch, Germany) according to the manufacturer's protocol. Sections (4- $\mu \mathrm{m}$ thickness) were immunostained with a primary antibody against BAP1 (1:100, sc-28383, mouse monoclonal, Santa Cruz Biotechnology, Santa Cruz, CA, USA). BAP1 expression level was evaluated according to the extent of nuclear staining in the tumor cells. The staining was scored as negative (no expression or expression in $<10 \%$ of tumor cells) or positive (expression in $\geq 10 \%$ of tumor cells).

\section{Statistical analysis}

All of the statistical analyses were performed using SPSS ver. 24.0 (IBM Corp., Armonk, NY, USA). The relationships between the groups were compared using the chi-square test, Fisher exact test, or Student's t test. Cancer-specific survival (CSS) was defined as the time interval between the date of surgical resection

Table 1. Histological and clinical characteristics

\begin{tabular}{lc}
\hline Characteristic & Value $(\mathrm{n}=371)$ \\
\hline Tumor type & \\
Clear cell RCC & $300(80.9)$ \\
Chromophobe RCC & $26(7.0)$ \\
Papillary RCC, type 1 & $13(3.5)$ \\
Papillary RCC, type 2 & $23(6.2)$ \\
Others & $9(2.4)$ \\
Age (yr) & $60.0(13-90)$ \\
Sex & \\
Male & $246(66.3)$ \\
Female & $125(33.7)$ \\
Tumor size & $3.77(0.7-15)$ \\
WHO/ISUP grade (clear and papillary RCC) & \\
1 & $33(9.8)$ \\
2 & $160(47.6)$ \\
3 & $113(33.6)$ \\
4 & $30(8.9)$ \\
Chromophobe grade (chromophobe RCC) & \\
\hline 1 & $19(73.1)$ \\
2 & $7(26.9)$ \\
Vascular invasion ${ }^{a}$ & \\
Absent & $323(87.1)$ \\
Present & $48(12.9)$ \\
Tumor necrosis & \\
Absent & $219(84.2)$ \\
Present & $41(15.8)$ \\
Sarcomatoid feature & $350(94.3)$ \\
\hline Present & $36(9.7)$ \\
Abmph node metastasis & $74(19.9)$ \\
\hline Present & $3(0.8)$ \\
\hline
\end{tabular}

Values are presented as number (\%) or median (range). RCC, renal cell carcinoma; WHO, World Health Organization; ISUP, International Society of Urological Pathology.

avascular invasion includes microscopic tumor invasion into small or large vessels and gross renal vein tumor thrombus. 

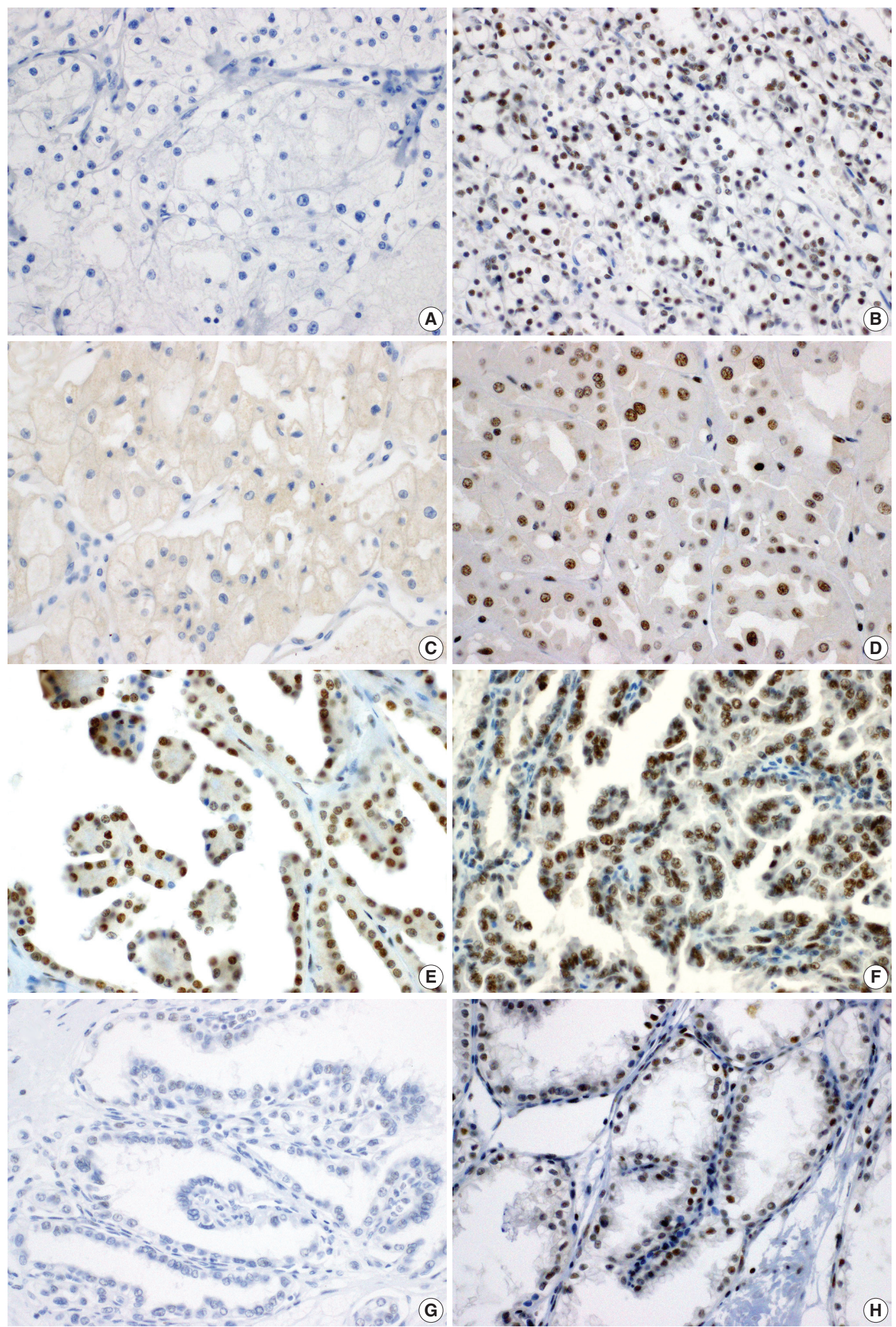

Fig. 1. Immunohistochemical staining of BRCA1-associated protein 1 in clear cell renal cell carcinoma (A, negative; $B$, positive), chromophobe renal cell carcinoma (RCC) (C, negative; D, positive), papillary RCC type 1 (E, positive), papillary RCC type 2 (F, positive), and clear cell papillary RCC (G, negative; $H$, positive). 
and the date of death due to RCC. Recurrence-free survival (RFS) was defined as the time interval between surgical resection and the date of any recurrence. The Kaplan-Meier method with the log-rank test and the Cox proportional hazard regression model were used for survival analyses. Two-sided p-values of $<.05$ were considered to indicate statistically significant differences.

\section{RESULTS}

\section{Patient characteristics}

The clinicopathological characteristics of the patients are summarized in Table 1. Among 371 RCC cases, the most common subtype was ccRCC (300 cases, 80.9\%). The other subtypes included in this study comprised 36 cases of papillary RCC (13 type 1 and 23 type 2 papillary RCC, 9.7\%), 26 cases of chromophobe RCC (7.0\%), four cases of clear cell papillary RCC, four cases of acquired cystic disease-associated RCC, and one case of collecting duct carcinoma. The age of the patients ranged from 13 to 90 years, with a median of 60 years. The median follow-up period for the patients in this study was 66 months (range, 0.1 to 167.6 months). Of the 371 patients, 11 (3.0\%) had metastatic disease at the time of initial diagnosis (i.e., nephrectomy), $30(8.1 \%)$ had experienced metastasis or relapse during the follow-up period, and 34 (9.2\%) had died due to RCC by

Table 2. Correlation between BAP1 expression and tumor type $(n=$ 371)

\begin{tabular}{lcc}
\hline & $\begin{array}{c}\text { Negative } \\
(\mathrm{n}=63)\end{array}$ & $\begin{array}{c}\text { Positive } \\
(\mathrm{n}=308)\end{array}$ \\
\hline Clear cell RCC & $56(18.7)$ & $244(81.3)$ \\
Chromophobe RCC & $6(23.1)$ & $20(76.9)$ \\
Papillary RCC type 1 & 0 & $13(100)$ \\
Papillary RCC type 2 & 0 & $23(100)$ \\
Clear cell papillary RCC & $1(25)$ & $3(75)$ \\
Acquired cystic disease-associated RCC & 0 & $4(100)$ \\
Collecting duct carcinoma & 0 & $1(100)$ \\
\hline
\end{tabular}

Values are presented as number (\%).

BAP1, BRCA1-associated protein 1; RCC, renal cell carcinoma.

Table 3. Correlations between BAP1 expression and clinicopathological features

\begin{tabular}{|c|c|c|c|c|c|c|}
\hline & \multicolumn{3}{|c|}{ Clear cell RCC $(n=300)$} & \multicolumn{3}{|c|}{ Chromophobe RCC $(n=26)$} \\
\hline & Negative $(n=56)$ & Positive $(n=244)$ & $p$-value & Negative $(n=6)$ & Positive $(n=20)$ & $p$-value \\
\hline Sex & & & .046 & & & .664 \\
\hline Male & $32(15.6)$ & $173(84.4)$ & & $3(27.3)$ & $8(72.7)$ & \\
\hline Female & $24(25.3)$ & $71(74.7)$ & & $3(20.0)$ & $12(80.0)$ & \\
\hline WHO/ISUP grade & & & .002 & & & \\
\hline 1 & 0 & $25(100)$ & & & & \\
\hline 2 & $20(14.1)$ & $122(85.9)$ & & & & \\
\hline 3 & $27(25.7)$ & 78 (74.3) & & & & \\
\hline 4 & $9(32.1)$ & $19(67.9)$ & & & & \\
\hline Chromophobe grade & & & & & & .146 \\
\hline 1 & & & & $6(31.6)$ & $13(68.4)$ & \\
\hline 2 & & & & 0 & $7(100)$ & \\
\hline Vascular invasion & & & .664 & & & .231 \\
\hline Absent & 49 (19.1) & $203(80.9)$ & & $5(20.0)$ & $20(80.0)$ & \\
\hline Present & $7(16.3)$ & $36(83.7)$ & & $1(100)$ & 0 & \\
\hline Tumor necrosis & & & .437 & & & \\
\hline Absent & 28 (16.8) & $139(83.2)$ & & $6(23.1)$ & 20 (76.9) & \\
\hline Present & $8(22.2)$ & $28(77.7)$ & & & & \\
\hline Sarcomatoid feature & & & .346 & & & \\
\hline Absent & $51(18.1)$ & $231(81.9)$ & & $6(23.1)$ & $20(76.9)$ & \\
\hline Present & $5(27.8)$ & $13(72.2)$ & & & & \\
\hline pT categorya & & & .037 & & & .606 \\
\hline pT1 & 35 (16.9) & $172(83.1)$ & & $3(18.8)$ & $13(81.3)$ & \\
\hline pT2 & $10(37.0)$ & 17 (63.0) & & $2(40.0)$ & $3(60.0)$ & \\
\hline рT3/4 & $11(16.7)$ & 55 (83.3) & & $1(20.0)$ & $4(80.0)$ & \\
\hline pN category ${ }^{a}$ & & & .899 & & & $>.999$ \\
\hline pNO & 55 (18.7) & 239 (81.3) & & $6(24.0)$ & 19 (76.0) & \\
\hline $\mathrm{pN} 1$ & $1(16.7)$ & $5(83.3)$ & & 0 & $1(100)$ & \\
\hline
\end{tabular}

Values are presented as number (\%).

BAP1, BRCA1-associated protein 1; RCC, renal cell carcinoma; WHO, World Health Organization; ISUP, International Society of Urological Pathology. ${ }^{a}$ AJCC eighth edition. 
the time of analysis.

\section{BAP1 expression and tumor type evaluation}

A total of 371 successfully stained cases with adequate clinical follow-up were classified as either BAP1 negative ( $\mathrm{n}=63,17.0 \%)$ or BAP1 positive $(n=308,83.0 \%)$. Representative images of BAP1 staining are shown in Fig. 1. Loss of BAP1 expression was frequently observed in ccRCC $(18.7 \%)$ and chromophobe RCC $(23.1 \%)$, while we failed to observe BAP1 expression loss in other renal tumor subtypes, including papillary RCC, acquired cystic disease-associated RCC, and collecting duct carcinoma. In clear cell papillary RCC, one case showed loss of BAP1 expression (Table 2).

\section{BAP1 expression and clinicopathological features of patients with ccRCC and chromophobe RCC}

In ccRCC, loss of BAP1 expression was significantly associated with female sex $(\mathrm{p}=.046)$ and high WHO/ISUP grade $(\mathrm{p}=$ .002). Furthermore, BAP1 expression loss was more frequent in pT2 than in pT1 category (Table 3). Other clinicopathological parameters such as vascular invasion, tumor necrosis, sarcomatoid feature, and lymph node status showed no significant association with BAP1 expression (Table 3).

In chromophobe RCC, no significant correlation was observed between BAP1 expression and clinicopathological parameters (Table 3).

\section{BAP1 expression and survival in ccRCC}

Of 300 patients with ccRCC, 10 (3.3\%) had metastatic disease at the time of initial diagnosis (i.e., nephrectomy), 23 (7.7\%) had experienced metastasis or relapse during the follow-up period, and $26(8.7 \%)$ had died due to RCC by the time of analysis.

Kaplan-Meier analysis and Cox regression analysis were applied to evaluate the prognostic value of BAP1 loss. Univariate analysis revealed that high WHO/ISUP grade, vascular invasion, tumor necrosis, sarcomatoid feature, high pT category, and lymph node metastasis predicted a poor outcome in ccRCC (Table 4). However, BAP1 expression showed no association with CSS and RFS (Fig. 2A, B). Even in cases with low pT (pT1/2) ccRCC, loss of BAP1 expression showed no statistically significant correlation with CSS and RFS (Fig. 2C, D).

\section{DISCUSSION}

In this study, we demonstrated the loss of BAP1 nuclear expression in chromophobe RCC and clear cell papillary RCC as well as ccRCC. Furthermore, the loss of BAP1 nuclear expression was associated with adverse clinicopathological features such as

Table 4. Univariate Cox regression analyses for cancer-specific survival and recurrence-free survival in patients with clear cell RCC

\begin{tabular}{|c|c|c|c|c|c|c|}
\hline \multirow{2}{*}{ Variable } & \multicolumn{3}{|c|}{ Cancer-specific survival } & \multicolumn{3}{|c|}{ Recurrence-free survival } \\
\hline & $\mathrm{HR}$ & $95 \% \mathrm{Cl}$ & $p$-value & $\mathrm{HR}$ & $95 \% \mathrm{Cl}$ & $p$-value \\
\hline \multicolumn{7}{|l|}{ BAP1 } \\
\hline Positive vs negative & 1.076 & $0.405-2.859$ & .884 & 1.125 & $0.462-2.740$ & .795 \\
\hline \multicolumn{7}{|l|}{ Sex } \\
\hline Female vs male & 2.140 & $0.807-5.676$ & .118 & 1.879 & $0.814-4.334$ & .139 \\
\hline \multicolumn{7}{|l|}{ WHO/ISUP grade } \\
\hline $1-2$ & 1 & & & 1 & & \\
\hline 3 & 3.401 & $1.138-10.165$ & .028 & 2.484 & $0.997-6.185$ & .051 \\
\hline 4 & 20.883 & $7.323-59.550$ & $<.001$ & 16.202 & $6.749-38.890$ & $<.001$ \\
\hline \multicolumn{7}{|l|}{ Vascular invasion } \\
\hline Absent vs present & 9.386 & $4.306-20.460$ & $<.001$ & 8.219 & $4.130-16.355$ & $<.001$ \\
\hline \multicolumn{7}{|l|}{ Tumor necrosis } \\
\hline Absent vs present & 18.216 & $5.713-58.079$ & $<.001$ & 16.980 & $5.953-48.428$ & $<.001$ \\
\hline \multicolumn{7}{|l|}{ Sarcomatoid feature } \\
\hline Absent vs present & 13.933 & $6.283-30.896$ & $<.001$ & 10.823 & 5.103-22.957 & $<.001$ \\
\hline \multicolumn{7}{|l|}{ pT category } \\
\hline pT1 & 1 & & & 1 & & \\
\hline pT2 & 6.298 & $1.409-28.145$ & .016 & 8.249 & $2.387-28.509$ & .001 \\
\hline pT3 and pT4 & 18.200 & $6.185-53.557$ & $<.001$ & 18.733 & 7.103-49.404 & $<.001$ \\
\hline \multicolumn{7}{|l|}{ Lymph node metastasis } \\
\hline Absent vs present & 32.885 & $10.417-103.815$ & $<.001$ & 18.940 & $6.203-57.828$ & $<.001$ \\
\hline
\end{tabular}

RCC, renal cell carcinoma; HR, hazard ratio; Cl, confidence interval; BAP1, BRCA1-associated protein 1; WHO, World Health Organization; ISUP, International Society of Urological Pathology. 

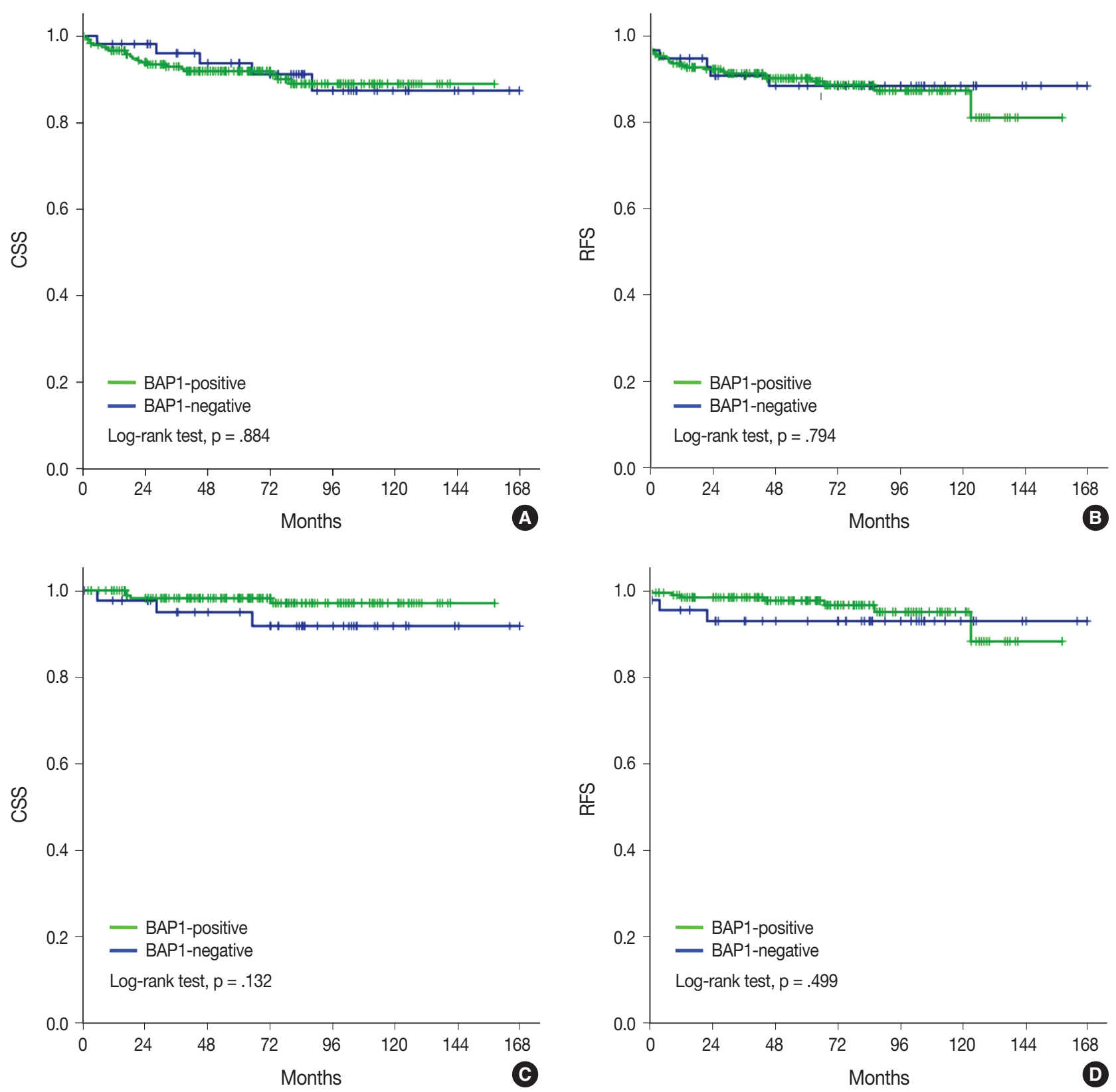

Fig. 2. Kaplan-Meier analysis of cancer-specific survival (CSS) and recurrence-free survival (RFS) in clear cell renal cell carcinoma (A, CSS; B, RFS) and pT1/2 ccRCC (C, CSS; D, RFS). BAP1, BRCA1-associated protein 1.

high WHO/ISUP grade in ccRCC but showed no relationship with CSS or RFS in patients with ccRCC.

The Cancer Genome Atlas (TCGA) research network recently reported the molecular characterization of RCC and included 488 ccRCC, 160 papillary type 1 RCC, 70 papillary type 2 RCC, and 81 chromophobe RCC. BAP1 mutation, a chromatin remodeling gene mutation, was reported in ccRCC (11.0\%) and papillary RCC (5.6\%) but not in chromophobe $\mathrm{RCC} .^{20} \mathrm{BAP1}$ mutation was also shown to be correlated with decreased survival in ccRCC. $^{20}$

The ratio of BAP1 loss in the present study (18.7\%) was similar to that reported in previous studies. ${ }^{21,22}$ We also found that the loss of BAP1 expression was significantly common in female sex $(\mathrm{p}=.046)$. BAP1 mutation was more frequent in female patients as per TCGA data. ${ }^{20,23}$ We observed that BAP1 nuclear expression loss was associated with high WHO/ISUP grade in ccRCC and showed no correlation with CSS and RFS. In several studies, loss of BAP1 expression served as an independent 
marker of prognosis in patients with ccRCC and low-grade ccRCC. ${ }^{24,25}$ On the other hand, in other studies, no significant association was reported between BAP1 loss and CSS or RFS, although BAP1 loss significantly correlated with poor clinicopathological parameters. ${ }^{16,21}$ Differences in the prognostic associations may be related to differences in cohorts among studies. Our cohort had relatively low-grade RCC and a short follow-up period; therefore, overall cancer-specific death rate was lower than that recorded in the previous TGCA report (8\% vs $33 \%){ }^{20}$

Among non-ccRCC, papillary RCC, chromophobe RCC, clear cell papillary RCC, acquired cystic disease-associated RCC, and collecting duct carcinoma were evaluated for BAP1 expression. We observed the loss of BAP1 expression in $23.1 \%$ of chromophobe RCC (6/26) cases and in one clear cell papillary RCC case. No significant association was detected between BAP1 expression and adverse clinicopathological parameters in chromophobe RCC. Unfortunately, the number of patients with chromophobe RCC and clear cell papillary RCC was too small to evaluate proper clinical relevance. In addition, during the follow-up period, one patient died due to chromophobe RCC; therefore, survival analysis could not be performed. An additional analysis is needed to further elucidate the role of BAP1 and the relationship between loss of BAP1 expression in IHC and BAP1 mutation in chromophobe RCC and clear cell papillary RCC.

In conclusion, we revealed that BAP1 expression is associated with high WHO/ISUP grade in patients with ccRCC and that BAP1 expression loss is also observed in chromophobe RCC and clear cell papillary RCC. Further studies are needed to assess larger cohorts and associated pathological features.

\section{ORCID}

Su-Jin Shin: https://orcid.org/0000-0001-9114-8438

\section{Conflicts of Interest}

No potential conflict of interest relevant to this article was reported.

\section{Acknowledgments}

This work was supported by the research fund of Hanyang University (HY-2016).

\section{REFERENCES}

1. Rini BI, Campbell SC, Escudier B. Renal cell carcinoma. Lancet 2009; 373: 1119-32.
2. Dalgliesh GL, Furge K, Greenman C, et al. Systematic sequencing of renal carcinoma reveals inactivation of histone modifying genes. Nature 2010; 463: 360-3.

3. Guo G, Gui Y, Gao S, et al. Frequent mutations of genes encoding ubiquitin-mediated proteolysis pathway components in clear cell renal cell carcinoma. Nat Genet 2011; 44: 17-9.

4. Varela I, Tarpey P, Raine K, et al. Exome sequencing identifies frequent mutation of the SWI/SNF complex gene PBRM1 in renal carcinoma. Nature 2011; 469: 539-42.

5. Hakimi AA, Ostrovnaya I, Reva B, et al. Adverse outcomes in clear cell renal cell carcinoma with mutations of 3 p21 epigenetic regulators BAP1 and SETD2: a report by MSKCC and the KIRC TCGA research network. Clin Cancer Res 2013; 19: 3259-67.

6. Peña-Llopis S, Vega-Rubín-de-Celis S, Liao A, et al. BAP1 loss defines a new class of renal cell carcinoma. Nat Genet 2012; 44: 751-9.

7. White AE, Harper JW. Cancer: emerging anatomy of the BAP1 tumor suppressor system. Science 2012; 337: 1463-4.

8. Bhattacharya S, Hanpude P, Maiti TK. Cancer associated missense mutations in BAP1 catalytic domain induce amyloidogenic aggregation: a new insight in enzymatic inactivation. Sci Rep 2015; 5: 18462.

9. Murali R, Wiesner T, Scolyer RA. Tumours associated with BAP1 mutations. Pathology 2013; 45: 116-26.

10. Rai K, Pilarski R, Cebulla CM, Abdel-Rahman MH. Comprehensive review of BAP1 tumor predisposition syndrome with report of two new cases. Clin Genet 2016; 89: 285-94.

11. Harbour JW, Onken MD, Roberson ED, et al. Frequent mutation of BAP1 in metastasizing uveal melanomas. Science 2010; 330: 1410-3.

12. Bott M, Brevet M, Taylor BS, et al. The nuclear deubiquitinase BAP1 is commonly inactivated by somatic mutations and 3p21.1 losses in malignant pleural mesothelioma. Nat Genet 2011; 43: 668-72.

13. Andrici J, Sheen A, Sioson L, et al. Loss of expression of BAP1 is a useful adjunct, which strongly supports the diagnosis of mesothelioma in effusion cytology. Mod Pathol 2015; 28: 1360-8.

14. Koopmans AE, Verdijk RM, Brouwer RW, et al. Clinical significance of immunohistochemistry for detection of BAP1 mutations in uveal melanoma. Mod Pathol 2014; 27: 1321-30.

15. Oka S, Inoshita N, Miura Y, et al. The loss of BAP1 protein expression predicts poor prognosis in patients with nonmetastatic clear cell renal cell carcinoma with inferior vena cava tumor thrombosis. Urol Oncol 2018; 36: 365.e9-e14.

16. Minardi D, Lucarini G, Milanese G, Montironi R, Di Primio R. Prognostic role of BAP1 in pT1 clear cell carcinoma in partial nephrectomy specimens. Virchows Arch 2017; 471: 99-105.

17. Eble JN, Sauter G, Epstein JI, Sesterhenn IA. World Health Organization classification of tumors: pathology and genetics of tumours 
of the urinary system and male genital organs. Lyon: IARC Press, 2016.

18. Delahunt B, Cheville JC, Martignoni G, et al. The International Society of Urological Pathology (ISUP) grading system for renal cell carcinoma and other prognostic parameters. Am J Surg Pathol 2013; 37: 1490-504.

19. Paner GP, Amin MB, Alvarado-Cabrero I, et al. A novel tumor grading scheme for chromophobe renal cell carcinoma: prognostic utility and comparison with Fuhrman nuclear grade. Am J Surg Pathol 2010; 34: 1233-40.

20. Ricketts CJ, De Cubas AA, Fan H, et al. The Cancer Genome Atlas comprehensive molecular characterization of renal cell carcinoma. Cell Rep 2018; 23: 3698.

21. Kim SH, Park WS, Park EY, et al. The prognostic value of BAP1, PBRM1, pS6, PTEN, TGase2, PD-L1, CA9, PSMA, and Ki-67 tissue markers in localized renal cell carcinoma: a retrospective study of tissue microarrays using immunohistochemistry. PLoS One 2017; 12: e0179610.

22. da Costa WH, da Cunha IW, Fares AF, et al. Prognostic impact of concomitant loss of PBRM1 and BAP1 protein expression in early stages of clear cell renal cell carcinoma. Urol Oncol 2018; 36: 243.e1e8.

23. Ricketts CJ, Linehan WM. Gender specific mutation incidence and survival associations in clear cell renal cell carcinoma (CCRCC). PLoS One 2015; 10: e0140257.

24. Joseph RW, Kapur P, Serie DJ, et al. Loss of BAP1 protein expression is an independent marker of poor prognosis in patients with low-risk clear cell renal cell carcinoma. Cancer 2014; 120: 1059-67.

25. Kapur P, Christie A, Raman JD, et al. BAP1 immunohistochemistry predicts outcomes in a multi-institutional cohort with clear cell renal cell carcinoma. J Urol 2014; 191: 603-10. 